\title{
A Sensor-fusion Framework for Queue-less Check-out in Retail
}

\author{
Anil Kumar Shukla, PhD* Tanmoy Gayen ${ }^{\dagger}$ \\ Atos Syntel
}

\begin{abstract}
The brick-and-mortar retail supermarket experience of customers is on the verge of another drastic change, this time enabled by advances in smart technologies. The deployment of queue-less check-out systems has the potential to drive customer satisfaction in years to come. Implementation of such systems require deployment of multiple smart sensor systems based on varied technologies and their seamless integration through sensor-fusion. This paper attempts to break-down these complex systems through a generalized framework. A sensor-fusion based framework is presented which can be used in designing a queue-less checkout system in a retail setting. The frame-work is composed of several independent sensor platforms. Different sensor-technologies can be used in each of the components of the framework either individually or in combination. A brief overview of a number of available sensor-technologies is provided.There are a few enterprises who have successfully commercialized queue-less retail check-out systems, however, very limited information is available in literature on how these systems actually work. Though considerable work has been done in different areas of sensor-technology development, research in ways to integrate multiple systems is still limited. The authors hope that this framework will benefit a wider set of researchers and businesses in designing and implementing such a system.
\end{abstract}

Keywords: sensor-fusion, queue-less, checkout, retail, in-store

\section{Introduction}

The retail sector has been going through a major transformation for quite a few years now. Starting with the advent of online retail, the digital wave has brought a great number of disruptive changes in this sector. The age-old brick-and-mortar retail businesses have been at the center of the storm of changes brought in by this new-age digital phenomena. The sense of existential threat from the emerging technology-driven business models earlier, has gradually turned into cautious optimism for the future where the digital innovations are seen as enablers for the brick-and-mortar business model. It is clear that the brick-and-mortar businesses are not going extinct but coming back stronger. This becomes more evident when we see online businesses like Amazon, Warby Parker, Bonobos, Casper among others opening brick-and -mortar stores.

However, to reap the rewards of this new digital world the brick-and-mortar business model needs to adapt to the reality that the consumer has become more powerful. With the availability of innumerable choices and information at their fingertips the retail consumer has the power to dictate in today's world [3]. Gone are the days where convenience of getting a large selection under one roof was the unique selling proposition. As more and more businesses are realizing that consumers, above all else, are increasingly focusing on the experience when choosing to go into a brick-and-mortar business, enhancing the in-store customer-experience has become a point of major discussion in the industry.

The in-store customer-experience is a holistic construct and involves a number of responses from the customer in cognitive, affective, emotional, social and physical $[4,5]$ contexts. Enhanced customer experience can be achieved by affecting one or more of the areas mentioned above. Creating a differentiated in-store experience which affects the cognitive aspect, is, to some extent dependent on the kind of goods the store sells and hence cannot be easily altered. The other aspects like the affective, emotional and social aspects are being manipulated more widely and successfully by businesses. For example, Montreal-based men's retailer Frank \& Oak are offering an in-store barber-shop or retailers like Saks Fifth Avenue, Coach, Bonobos etc. better-equipping sales associates through the provision of mobile technology-enabled tablets to assist customers [2]. The queue-less check-out experience which is the primary focus of this paper affects primarily the physical responses of the cus-

Authors declare no conflict of interest. Both authors have made equal contribution in creating this paper.

Address (both authors): Syntel Private Limited, SEZ, Plot no: B1/B2, Talawade Software Park, Talawade, Pune 411062, India

*email: shuklaks@gmail.com

†Corresponding author,email: tanmoygayen01@gmail.com 
tomer. Through a considerable saving in terms of time spent in the check-out queue, a proper implementation of this system, would results in positive customer-experience.

The idea of queue-less check-out is in fact borrowing a page out of the online marketing platforms and fitting it right into the brick-and-mortar setting. Though not new, only a few players have been successful in implementing it in a way that truly enhances the customer experience. The most widely known and perhaps the most seamless is AmazonGo. However, other than a few bits and pieces of information there is no comprehensive information available on how such systems work. Being developed on advancements, some of which are too new, there is a lack of information on such systems in literature as well. In this paper we propose a framework on how such a system can be built. Then we explore a number of technologies, some emerging and some already mature, which can be used as components in the proposed framework.

\section{Background}

The concept of enhancing customer-experience through the provision of super-market floor assistance of various kinds, not just limited to queue-less check-out, through digital innovations is not new, a number of companies have attempted the same since early 2000s [1]. Newer companies like ShelfX or Zebra Technologies (makers of SmartLens) have achieved technology solutions which are very similar.

However, none of the attempts were able to or directed towards completely by-passing the checkout system except AmazonGo. Since this is the best example of queue-less check-out in retail at present, it is important to have a slightly closer look at it. At the time of writing this article, Amazon has opened 5 AmazonGo stores [6]. The stores by and large are medium sized and generally stock food (both packed and prepared), beverages (including alcohol) and grocery essentials. Customers need to install the AmazonGo App on their cell phones where some basic personal information needs to be submitted including credit card for charging. Once installed and opened the application displays a QR code which needs to be swiped on a code reader enabled doorway while entering the shop. Once inside, customers can browse, peruse and take items as they please and simply walk out of the shop. After a while the cart gets calculated and receipt sent to the customer's cell phone. At the moment though, there is a limit to the number of customers who can enter the shops at any given moment and queues do form at the entry rather than during check-out, we argue that this is a limitation of the scale of implementation rather than the concept and hence can be mitigated. The other limitation is that once out of the shop, in case one does not require an item, there is currently no way to return it back after going back inside the shop. In terms of the technology used, Amazon has not gone beyond disclosing the usage of "computer vision, sensor fusion, and deep learning" [6].

\section{The Conceptual Framework}

In this section, we propose a framework which can be used to create a system that by-passes the physical check-out process. The framework consists of a number of elements or components each of which specializes in a particular task. The outputs of these independent elements are combined at a later stage. From a technology perspective each of these elements can be implemented using a number of different technologies some of which are comparatively new and some are quite mature. The different technologies that can be used for each of these elements will be discussed in the next section. As shown in Figure 1, the framework that we propose has three primary elements. The first one is the Customer Tracking System, the sole purpose of which is to track the customer through his or her shopping experience inside the shop. The second one is the Inventory Tracking System, which as the name suggests is concerned with tracking the quantity of items in stock. The third intelligent system is the Cart Calculation System, it takes the outputs of both the other two systems and reconciles them to calculate the customer cart.

\subsection{Customer Tracking System}

The Customer Tracking System (CTS) is composed of two separate sub-components viz. the Customer Location Tracking system and the Customer Action Tracking system. The Customer Location Tracking System (CTS-L) has 2 objectives:

- Customer Identification: When a customer walks inside the store, this system detects and identifies the customer. Further, it fetches relevant information (along with any unique customer key) from the customer database. A pre-requisite of the entire framework is that the customers already exist in the customer database (for example, in case of AmazonGo a customer gets on the database while installing the app). All the actions of the customer inside the store during the curren$\mathrm{t}$ visit are tagged and stored appropriately in the database against the already existing customer record.

- Customer Location Tracking: Once inside the store, the customer will usually explore var- 
ious shelves by moving around the shop. Since a customer can only pick-up items from a shelf which are within a particular distance from his or her location, it is important to track the customer's location throughout the visit and store the information. Finally, once the customer walks out of the store this system is responsible for tracking the same and logging end of a visit which will trigger a host of other actions downstream. These kinds of systems are known as internal positioning systems (IPS).

The CTS will output a log which will capture all the customers locations within the store at a given point of time along with the timestamp. This data can be enriched using different ways, for example including a simple flag-based scheme to capture the customer's proximity to individual shelves as shown in Figure 2.

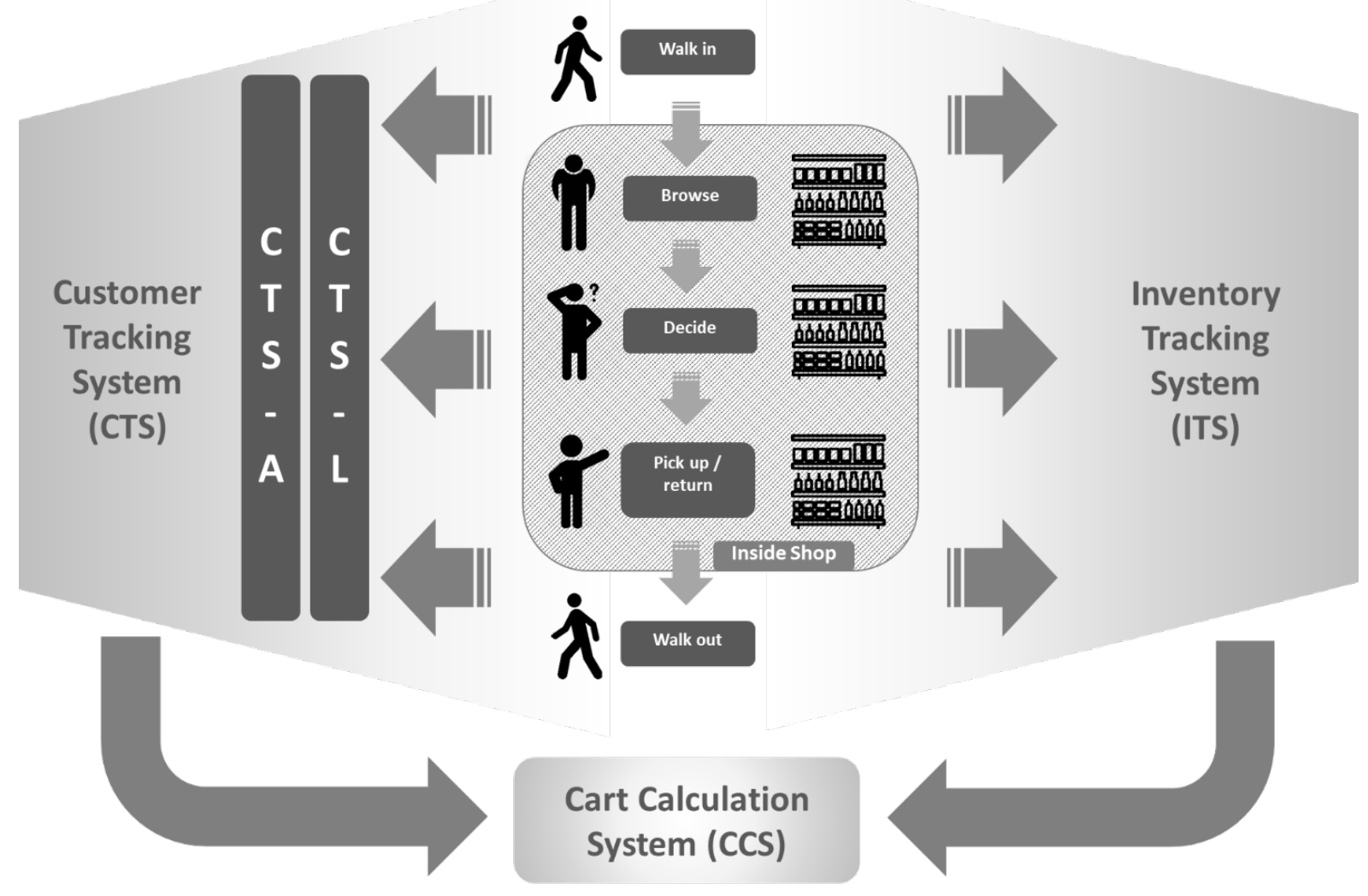

Figure 1: The proposed framework

\begin{tabular}{|c|c|c|c|c|c|c|c|c|}
\hline \multirow{2}{*}{ Time Stamp } & \multirow{2}{*}{ Customer ID } & \multicolumn{2}{|c|}{ Raw Location } & \multicolumn{5}{|c|}{ Shelf Vicinity Flags } \\
\hline & & X_Co-ord & Y_Co-ord & Item_1 & Item_2 & Item_3 & $\ldots \ldots$ & Item_n \\
\hline 15-01-2618 69:31:10 & 956431 & 574 & 733 & $\mathbf{1}$ & 1 & $\mathbf{B}$ & -- & $\mathbf{B}$ \\
\hline 15-01-2618 69:31:10 & 245786 & 769 & 112 & $\mathbf{\theta}$ & $\mathbf{\theta}$ & $\mathbf{1}$ & -- & $\mathbf{\theta}$ \\
\hline 15-01-2618 69:31:10 & 359563 & 928 & 636 & $\boldsymbol{\theta}$ & $\boldsymbol{\theta}$ & $\mathbf{\theta}$ & -- & $\mathbf{\theta}$ \\
\hline 15-01-2618 69:31:10 & 315635 & $\mathbf{2 9}$ & 101 & $\boldsymbol{\theta}$ & $\boldsymbol{\theta}$ & $\mathbf{\theta}$ & -- & $\mathbf{1}$ \\
\hline
\end{tabular}

Figure 2: Sample CTS-L Output data. 
The Customer Action Tracking System (CTSA) on the other hand looks for actions of interest that the customer performs once inside the store. From a physical perspective, broadly all customer actions inside the store can be categorized into the following:

- Moving

- Standing

- Taking or returning items ${ }^{1}$

This sub-system tracks these actions and creates a record of the actions taken with time-stamp. Clearly the last action defined above is of most importance, so an ideal system would get activated only when the customer takes or returns an item and would record the details of the action with the timestamp. Otherwise it will only log actions at pre- specified intervals. Figure 3 shows a sample log from CTS-A.

\subsection{Inventory Tracking System}

The Inventory Tracking System (ITS) is an automatic inventory maintenance system. If the shop stocks n different items on it's shelves, the ITS will monitor quantity of each of these $n$ items at regular intervals and maintain a log file. An ideal system would be one in which the monitoring mechanism is triggered when it detects a change in inventory, however a simpler mechanism which monitors continuously based on a pre-determined time interval will also be fine given the interval is small enough. A sample log generated by the ITS is shown in Figure 4.

\begin{tabular}{|c|c|c|c|c|}
\hline \multirow{2}{*}{ Time Stamp } & \multirow{2}{*}{ Customer ID } & \multicolumn{3}{|c|}{ Customer Actions } \\
\hline & & Move & Stand & |Take/Return \\
\hline 15-01-2018 69:31-85 & 956431 & $\mathbf{1}$ & $\boldsymbol{\theta}$ & $\mathbf{B}$ \\
\hline 15-01-2818 69:31585 & 245786 & $\boldsymbol{\theta}$ & $\mathbf{1}$ & E \\
\hline 15-01-2818 69:31585 & 359563 & $\boldsymbol{\theta}$ & $\mathbf{1}$ & $\boldsymbol{\theta}$ \\
\hline 15-01-2818 69:31:85 & 315635 & $\mathbf{1}$ & - & - \\
\hline 15-01-2818 69:31:18 & 956431 & $\mathbf{1}$ & $\boldsymbol{\theta}$ & B \\
\hline 15-01-2818 69:31:18 & 245786 & $\mathbf{1}$ & $\boldsymbol{\theta}$ & $\mathbf{1}$ \\
\hline $15-01-2818$ 69:31:18 & 359563 & $\mathbf{\theta}$ & $\mathbf{1}$ & B \\
\hline 15-01-2818 69:31=18 & 315635 & $\boldsymbol{\theta}$ & $\boldsymbol{\theta}$ & B \\
\hline 15-01-2818 69:31:15 & 956431 & $\mathbf{1}$ & 日 & E \\
\hline 15-01-2818 69:31:15 & 245786 & $\boldsymbol{\theta}$ & $\mathbf{1}$ & $\mathbf{\theta}$ \\
\hline 15-01-2818 69:31:15 & 359563 & $\boldsymbol{\theta}$ & $\mathbf{1}$ & $\mathbf{\theta}$ \\
\hline 15-01-2618 69:31:15 & 315635 & $\mathbf{1}$ & 日 & $\boldsymbol{\theta}$ \\
\hline
\end{tabular}

Figure 3: Sample CTS-A Output data.

\begin{tabular}{|c|c|c|c|c|c|}
\hline \multirow{2}{*}{ Time Stamp } & \multicolumn{5}{|c|}{ Inventory } \\
\hline & Item_1 & Item_2 & Item_3 & $\ldots \ldots$ & Item_n \\
\hline 15-81-2818 69:31-13 & 124 & 56 & 73 & & $\boldsymbol{\theta}$ \\
\hline 15-01-2818 69:31:45 & 124 & 56 & 7 & & $\boldsymbol{\theta}$ \\
\hline 15-01-2818 69:31:07 & 124 & 56 & 73 & & $\theta$ \\
\hline 15-01-2818 69:31:69 & 124 & 56 & 7 & & $\boldsymbol{\theta}$ \\
\hline 15-01-2818 69:31:11 & 124 & 56 & $\pi$ & & $\theta$ \\
\hline
\end{tabular}

Figure 4: Sample ITS Output data.

\footnotetext{
${ }^{1}$ Both taking/returning items from/to shelves have been tagged together as from a physical action perspective, both entail similar body movements on the part of a customer. Though it is possible to further tag these actions separately and would definitely make the system more accurate, it is not necessary to do so.
} 


\subsection{Cart Calculation System}

This is a reconciliation system which combines the outputs of CTS and ITS to generate the final customer cart. In essence, this component is responsible for tying together two different sensor platforms (CTS \& ITS) and hence can be considered as the primary component designed around the core concept of sensor-fusion.

The Cart Calculation System (CCS) uses a simple logic of inventory change attribution: If $i t h$ inventory item count at time $\mathrm{t}$ differs from $t-1$ then look for customers in vicinity of ith inventory item during time interval $t-1$ and $t$. Amongst identified customers who satisfy above criteria look for customers who performed an action of interest during the same time interval. Match obtained by time-stamp with small allowance for deviation depending on system lag. Attribution problems arising from situations where multiple customers perform multiple actions of interest on the same inventory item in the same time interval can be minimized by reducing the time interval. The algorithm is described in detail below.

\section{Key Elements:}

- Let the store contain $m$ shelves, each containing different items. We denote the inventory of the $i t h$ shelf (or item) at time $t$ by $s_{i, t}$ and the entire inventory at time $t$ can be represented by a vector $S_{t}$ of size $m$.

- Each of the $\mathrm{m}$ shelves will have a specific area from which it is accessible to the customers. For simplicity we assume that all such areas can be represented by quadrilaterals. The accessible area for the ith shelf can be denoted by a set of co-ordinates: $x_{i, \min }, y_{i, \min }, x_{i, \max }, y_{i, \max }$. The entire set of such co-ordinates can be represented by a matrix $R$ of size $m * 4$.

- Let us assume that there are $n$ customers in the shop. Each of these customers will have three parameters of our interest: in-store location, current action and cart content. We assume that the location of customers at time $t$ is represented by a $n * 2$ matrix $L_{t}$, where $l_{i, 1, t} \& l_{i, 2, t}$ gives the $\mathrm{x}$ and $\mathrm{y}$ co-ordinates of the $i t h$ customer at time $t$ respectively. Similarly, a $n * 3$ (3 since we have defined only 3 simple actions in our framework) matrix $A_{t}$ where $a_{i, 1, t}, a_{i, 2, t} \& a_{i, 3, t}$ represent binary states pertaining to actions move, stand and take/return for the ith customer at time $t$. Finally the customer cart can be represented by $n * m$ matrix $C_{t}$ where element $c_{i, j, t}$ gives the number of $j t h$ item in the cart of $i t h$ customer at time $t$.

\section{Algorithm:}

- Continuously compare inventory current state with the preceding state.

- Let $D_{t}=S_{t-1}-S_{t}$, if all elements of $D_{t}$ are zeroes, then continue, else:

- For all such $d_{i, t}=! 0$, do the following:

* Compare $R$ with $L_{t}$ to find which customers were within accessible area of the ith item. Criteria: $\quad r_{i, 1} \leq l_{j, 1, t} \leq r_{i, 3} \quad$ AND $r_{i, 2} \leq l_{j, 2, t} \leq r_{i, 4}$

* In case more than 1 customers are identified to be in the accessible area, check for action in matrix $A_{t}$. If $a_{j, 3, t}==1$ then inventory item can be tagged to the $j t h$ customer, i.e. $\quad c_{j, i, t}=c_{i, j,(t-1)}+d_{i, t}$. Else, whichever customer is in the accessible area would be tagged with the inventory item.

\section{Technologies}

In this section we take a brief look at the different technologies that can be used in building the individual components of the system proposed in the previous section.

\subsection{Customer Tracking System}

Customer Location Tracking System: As pointed out previously, CTS-L is nothing but an IPS. This has been a subject of research for quite some time now and a number of different technologies are available to achieve this like WIFI[11, 12], RFID[14, 15], UWB[13], BLE[7, 8, 16] and NFC[9, 17]. A number of methodologies have also been developed which can be used on systems built on multiple technologies mentioned above.

In general, all these systems are based on a mobile beacon whose position is estimated using measurements from a set of fixed signal transceivers. The beacon sends out signals at fixed intervals which the transceivers detect. The position of the beacon is estimated using methodologies which can be broadly classified into two types - trilateration and fingerprinting [12]. In trilateration approach, each of the transceivers independently estimate the distance of the mobile beacon and the position is estimated as the region of overlap between evenly spaced transceivers. The methods for estimating the mobile beacon position can be based on signal characteristics like time of arrival (TOA/TDOA), angle or arrival (AOA), received signal strength (RSS) etc.[13]. The fingerprinting method is based on a 
pre-built database of a signal characteristic (usually RSSI) corresponding with each possible beacon location in the area of interest. The position is estimated in real-time by comparing the actual received signal characteristic with those in the database value.

It is important to recognize that performance of solutions based on different technologies will vary from case to case. Selection of the appropriate technologies for a particular implementation may depend on a number of factors such as:

- Hardware compatibility: Each of the technologies have specific hardware requirements that need to be deployed both inside the store (transceivers and computational hardware) as well as with the customer (beacon). Some of the hardware for technologies like WiFi, BLE and NFC are already present in most modern smartphones hence would not require additional deployment with the customer, though additional software may be needed to be installed (in the form of an App like in the case of AmazonGo). For other technologies like RFID and UWB additional hardware may be required to be deployed with the customer. Point to note is that in either case hardware will be required to be installed inside the store.

- Cost: The hardware associated for each of these technologies vary widely in cost. Further, the size of the area of interest, expected number of beacons (customers) to be tracked simultaneously, power requirements of the components used, also affect the final cost of the solution. For a business implementing a solution to positively improve customer experience, the cost of the solution can become one of the key considerations during decision.

- Accuracy: The accuracy of the system is another important factor, as accuracy of the different technologies can vary widely from few centimeters (NFC) to a few meters (WLAN). However, the notion that the implemented system has to have an accuracy in the order of centimeters is not always correct, the order of accuracy would depend of the store design. Intelligent store design can eliminate the need of a very accurate IPS. Further, in most cases, the accuracy of the same IPS can be improved by increasing the density of sensors.

Customer Action Tracking System: Human action tracking has also been an active area of research for a number of years. It has come under renewed focus with advancements in motion capture \& storage technologies, newer areas of application like gesture recognition and newer machine learning driven image processing methodologies.

In general, the proposed methodologies for human action recognition can be classified into three types: template matching, state-space approach and action-signal matching. The template matching approach converts sequence of images into static shape and recognizes actions by comparing the patterns with pre-classified sequences from a database. In the state-space approach, a probability density is calculated for each of different action sequence from every pose of human body by considering each pose as a state. A complete sequence can be thought of as a permutation of different states. A probability density function can be estimated for different state-permutations of the same action. Probability functions can then be used to recognize test sequences. Action-signal matching is a signal processing based methodology. Here, the problem is treated as a classification problem of temporal activity signals according to pre-labeled reference signals representing typical human actions. Xu et al. [29] and Wang et al. [27] present good surveys of different methodologies used for human action recognition currently in use.

New age advanced machine learning concepts like convolutional neural networks and recurrent neural networks have also been employed in this area and are generally accepted to give better results. Murad et al. [28] used Deep Recurrent neural networks and compared results with traditional machine learning - support vector machines (SVM) and k-nearest neighbors (KNN).

Like CTS-L, while deploying this system, hardware, cost and accuracy would be the key considerations, however, unlike CTS-L, the underlying technology would be somewhat same. The key components would be an image-capture platform (set of cameras), an image processing and action recognition system (which would be a data storage and compute system).

\subsection{Inventory Tracking System}

Inventory tracking is an area which probably predates all other automatic sensing problems associated with the entire queue-less retail check-out process. Solutions are available based on a number of technologies like RFID [18], weight-sensors [19, 22, 23], pegs and pushers[22]. Some solutions have been developed by sensing the level of light in the shelves[24].

Image recognition is another technology that can be used in building this solution. We were able to achieve greater than $90 \%$ accuracy in predicting the number of items on a virtual shelf image generated and randomly populated with items using a rudimentary python program. We had used a com- 
paratively simple convolutional neural network ( 8 convolution layers and 2 dense layers) to achieve this. However, true potential of image recognition is harnessed in solving more complicated problems like planogram compliance[20, 21, 26], which is a pre-requisite but not a direct component for a system like this or differentiating between very similar products (AmazonGo uses large visual codes which can be easily picked up by overhead cameras on prepared food items which have similar packaging and weight).

Some of these products are already commercialized to a level that plug-and-play components are available, for example ShelfX[23] whose systems are more focused on weight based sensors or Wiseshelf[24] which focusses on light-level detection on shelves.

\subsection{The concept of Sensor Fusion}

The accuracy and deployability of a particular technology-based sensor-solution will vary from one scenario to another, however to achieve a robust solution with acceptable accuracy, multiple technology-based sensors should be used in tandem. This concept of using different sensors and combining their output is the basic idea of sensor fusion. This idea is the basis of CCS in our framework.

Further, multiple types of sensors can be used together in the implementation of the same component of the framework. This concept is demostrated by Smowton et al. [7] where they combine face recognition with Bluetooth based position sensing to achieve better results. Commercially available products like SmartLens (Zebra technologies) also make use of a combination of multiple types of sensors. Finally AmazonGo has specifically mentioned the usage of sensor fusion (albeit they did not mention the different types) in their solution.

\section{Conclusion}

A queue-less check-out system described in this paper has a certain limitations, for example, as mentioned earlier, planogram compliance becomes a key pre-requisite for it to operate correctly. Another key pre-requisite is the customer database, which needs to be in place before the customer can enter the shop-floor. Further, the maximum number of customers that can be allowed inside the store simultaneously would be limited for accurate operation and dependent on the type of technologies used.

Again, while considering the deployment of a solution like this, one of the key considerations of the businesses should around what they sell and who their customers are. For example a retailer who sells luxury items may derive better customer satisfaction with well-trained store attendants who can deliver a more personalized experience rather than a queue-less check-out solution.

Experiencing a novel concept like queue-less checkout in a retail store, presented with sophistication and finesse like in case of AmazonGo can be overwhelming. Visualizing the underlying system$\mathrm{s}$, architecture and technologies may seem difficult and complicated. In this paper we have attempted to break down the underlying systems into the proposed conceptual framework and highlighted some of the technology solutions that can be used to implement such a solution. We hope that with the current advances in technology, as it becomes more available and affordable, even smaller enterprises with less resources will be able to provide a similar experience to their customers.

\section{References}

[1] Marc Janssens and Paul Burel, Retail Business Unit, Fujitsu America Inc. Market Insight Amazon Go and the Future of Brick-and-Mortar Retail

[2] Jesse Linklater and Anne Mulvenna. Future of Retail: In Stores Now

[3] Shaping the Future of Retail for Consumer Industries, World Economic Forum Insight Report, Jan 2017

[4] Peter C. Verhoef, Katherine N. Lemon, A. Parasuraman, Anne Roggeveen, Michael Tsiros, and Leonard A. Schlesinger. Customer experience creation : Determinants, dynamics and management strategies. 2009.

[5] Juan Bustamante and Natalia Rubio. In store customer experience: a first approximation in the construction of a scale. 012017.

[6] AmazonGo https: //www . amazon.com/ b? ie $=U T F 8 \&$ node $=16008589011$

[7] Christopher Smowton, Jacob R. Lorch, David Molnar, Stefan Saroiu, and Alec Wolman. Seamless customer identification. 2013.

[8] Jun-Ho Huh and Kyungryong Seo. An indoor location-based control system using bluetooth beacons for iot systems. In Sensors, 2017.

[9] Busra Ozdenizci, Vedat Coskun, and Kerem Ok. Nfc internal: An indoor navigation system. In Sensors, 2015. 
[10] Jose Antonio Puertolas-Montañez, Adriana Mendoza-Rodriguez, and Ivan Sanz-Prieto. Smart indoor positioning/location and navigation: A lightweight approach. IJIMAI, 2:4350, 2013.

[11] Phongsak Prasithsangaree, Prashant Krishnamurthy, and Panos K. Chrysanthis. On indoor position location with wireless lans. In PIMRC, 2002.

[12] Binghao Li, James Salter, Andrew G. Dempster, and Chris Rizos. Indoor positioning techniques based on wireless lan. 2006.

[13] Abdulrahman Alarifi, AbdulMalik S. AlSalman, Mansour Alsaleh, Ahmad Alnafessah, Suheer Alhadhrami, Mai A. Al-Ammar, and Hend Suliman Al-Khalifa. Ultra wideband indoor positioning technologies: Analysis and recent advances $\dagger$. In Sensors, 2016.

[14] Samer S. Saad and Zahi S. Nakad. A standalone rfid indoor positioning system using passive tags. IEEE Transactions on Industrial Electronics, 58:1961-1970, 2011.

[15] Kevin Weekly, Han Zou, Lihua Xie, Qing-Shan Jia, and Alexandre M. Bayen. Indoor occupan$\mathrm{t}$ positioning system using active rfid deployment and particle filters. 2014 IEEE International Conference on Distributed Computing in Sensor Systems, pages 35-42, 2014.

[16] Vicente Cantón Paterna, Anna Calveras Augé, Josep Paradells Aspas, and María Alejandra Pérez Bullones. A bluetooth low energy indoor positioning system with channel diversity, weighted trilateration and kalman filtering. In Sensors, 2017.

[17] Wilson Sakpere, Nhlanhla Mlitwa, and Michael Adeyeye Oshin. Towards an efficient indoor navigation system: a near field communication approach. Journal of Engineering, Design and Technology, 15:00-00, 062017.
[18] Christian Decker, Uwe Kubach, and Michael Beigl. Revealing the retail black box by interaction sensing. In ICDCS Workshops, 2003.

[19] wiihey.com http://www.wiihey.com/en/ applications/smart-shelf-management-system.html

[20] Timothy E Chong and Idawati Bustan. Deep learning approach to planogram compliance in retail stores.

[21] Alessio Tonioni and Luigi di Stefano. Product recognition in store shelves as a sub-graph isomorphism problem. In ICIAP, 2017.

[22] NACS Magazine https://www . convenience.org/Media/ NACS-Magazine/2016/September2016/Feature5

[23] ShelfX http://www.shelfx.com/

[24] WiseShelf https://www.wiseshelf.com/

[25] Zebra Technologies SmartLens https://www.zebra.com/us/en/solutions/ retail-solutions/smartlens-for-retail.html

[26] Gül Varol and Ridvan Salih Kuzu. Toward retail product recognition on grocery shelves. 2014.

[27] Liang Wang, Weiming Hu, and Tieniu Tan. Recent developments in human motion analysis. Pattern Recognition, 36:585-601, 2003.

[28] Abdulmajid Murad and Jae-Young Pyun. Deep recurrent neural networks for human activity recognition. In Sensors, 2017.

[29] Xin Xu, Jinshan Tang, Xiaolong Zhang, Xiaoming Liu, Hong Zhang, and Yimin Qiu. Exploring techniques for vision based human activity recognition: Methods, systems, and evaluation. In Sensors, 2013. 